\title{
Emergency Message Dissemination System for Smartphones During Natural Disasters
}

\author{
Xian Wu, Maciej Mazurowski, Zhen Chen, Nirvana Meratnia \\ Electrical Engineering, Mathematics and Computer Science \\ University of Twente, the Netherlands \\ Email: \{x.wu,m.t.mazurowski,z.chen\}@ student.utwente.nl; \\ N.Meratnia@utwente.nl
}

\begin{abstract}
This paper revolves around the concept of utilizing the modern smartphone communication capabilities to transmit messages through an ad hoc network during a disaster, which renders the traditional cellular base station inaccessible. Due to dynamic and decentralized nature of the considered environment, epidemic algorithms present themselves as a suitable option for message dissemination. We consider a specific scenario and propose a modified epidemic routing protocol that could be useful during natural disasters. We develop a simulation tool using MATLAB to evaluate the influence of various factors on the performance and cost of our modified epidemic routing protocol in case of an emergency.
\end{abstract}

Index Terms-Epidemic Routing; Emergency Application; Smartphone; Opportunistic Network

\section{INTRODUCTION}

In ubiquitous computing, computers become a helpful but invisible force, assisting the user in meeting his or her needs without getting in the way [1]. A smartphone can be seen as a ubiquitous computing platform. Smartphone is a new generation mobile phone that offers increased computational and connectivity capabilities and is able to run complete operating systems that are used as a platform for application developers. Thus, a smartphone usually allows the user to install and run more advanced applications. According to a study by ComScore [2], over 45.5 million people in the United States out of 234 million total subscribers owned smartphones in 2010. In March 2011 Berg Insight reported that global smartphone shipments increased 74\% from 2009 to 2010 [3]. The popularity of smartphones has been unprecedentedly increased.

A smartphone is usually equipped with dedicated chips for logic processing (CPU), graphics processing (GPU) and communication components. In addition, smartphones are embedded with cameras and a wide variety of sensors and transducers. Last but not least, smartphones support various transmission technologies, including infrared, Bluetooth, WiFi and GPS. It is worth mentioning that the advanced and accurate input methods [4] and operation systems such as iOS and Android [5] [6] support ubiquitous computing's vision well.

We explore a potential application of smartphones in the case that natural disasters or other emergencies occur, which becomes frequent all around the world. A rescue system using the Android Technology is proposed in [7]. However, disasters often come along with the destruction of the local telecommunication infrastructure causing severe problems for rescue team. In this case, to re-establish communication between victims and outside world, sending the emergency messages through ad hoc wireless networks would be favorable.

In our application, no assumption is made with regard to the existence of a complete path between two nodes wishing to communicate. Any possible node can opportunistically be used as a next hop, provided brings the message closer to the final destination. These features match the concept of opportunistic networking. Therefore, the ad hoc network consisting of smartphones can be regarded as an opportunistic network.

We focus on this very topic and propose a feasible solution for emergency message dissemination. This paper is organized as follows. Firstly, section II defines the specific usage scenario. In section III, the wireless technologies used in our application are discussed. Section IV introduces basic principles of the proposed algorithm like message format and routing techniques. Section V and section VI demonstrate our simulation method and results. Section VII concludes the paper and looks into future work.

\section{USAGE SCENARIO}

Various cities around the world are vulnerable to natural hazards such as earthquake, hurricane and tsunami. These destructive hazards are capable of destroying base stations, making the GSM or UMTS network completely disabled. This is the main presupposition of our research and difference between ours and existing emergency communication solutions.

\section{A. Objective}

The objective is to realize a one-way communication in case of an emergency between a source smartphone and a destination in absence of operational base stations inside an emergency region. To achieve this goal, our idea is to take advantage of wireless communication of smartphones to establish an ad hoc network, broadcast and forward the message from the source to operational regions, where cellular architecture is available. 


\section{B. Roles}

There are three roles for smartphones in the described ad hoc network.

- Source

The message source could belong to victims trapped in a building/ruin or injured people in need of rescue. They are usually unable to move, therefore are static sources. The source smartphone can also belong to witnesses or reporters sending the real-time information out. Such node can be considered as dynamic sources.

- Destination

The final destination could be either an emergency center (police, red cross etc.) or a mobile user (the source's family or friend). From technical point of view, here we consider a nearby operational base station outside the disaster region as the destination.

- Intermediate Nodes

These are smartphones within the disaster region that participate in the message dissemination by virtue of their wireless capabilities. In our scenario, these capabilities are realized by Bluetooth or WiFi (IEEE 802.11). The motivation for our choice will be explained in section III. Since not all the smartphones support both WiFi and Bluetooth, here we classify them into three groups: mobiles that only support WiFi, only support Bluetooth and support both.

\section{Assumption}

To achieve our objective, using the ad hoc mode (rather than infrastructure mode because there is no Access Point) of 802.11 family protocol is the most suitable choice. However, as a matter of fact, the state-of-art smartphone operation systems do not support this mode very well. Hereby we assume that it is feasible to work in ad hoc mode through some software configuration or upgrades.

\section{WIRELESS TECHNIQUES}

Based on our scenario, we investigated the potential of four wireless technologies: 802.11, Bluetooth, Infrared and FM Radio Transmission. All other technologies such as ZigBee, satellite, Digital Radio Mondiale, Terrestrial Trunked Radio were discarded because of not being implemented in modern smartphones. After further investigation, we also removed Infrared and FM Radio Transmission from the list because of their insufficient ranges and not being popular transmission standards. This left us with just two technologies that are relatively widely implemented and provide satisfactory range. Nowadays, most smartphones are equipped with $802.11 \mathrm{~b}$ and Bluetooth 2.0/2.1, which has faster data rate than version 1.2.

\section{A. $W i F i$}

WiFi or IEEE 802.11 is a set of standards consisting of over-the-air modulation techniques that use the same basic protocol. Typical 802.11 usage scenarios are: data and voice access, ad hoc networking, cable replacement. The 802.11 family encompasses various versions like 802.11a, 802.11b, and $802.11 \mathrm{~g}$. Since the $802.11 \mathrm{~b}$ is the most popular version among mobile platforms, we select it to build our ad hoc network and to transmit the message.

\section{B. Bluetooth}

Bluetooth is a radio interface operating in the $2.4 \mathrm{GHz}$ frequency band that allows mobile devices to communicate in a wireless manner by creating a relatively short-range (10 meters) ad hoc networks, which was created mostly as a cable replacement technology [8]. Each device can connect with seven other devices per Bluetooth network cell - a piconet. In addition, one device can belong to several piconets.

TABLE I

A COMPARISON OF BLUETOOTH AND 802.11B SPECIFICATIONS

\begin{tabular}{l|rr}
\hline & Bluetooth 2.0 & $802.11 \mathrm{~b}$ \\
\hline frequency band & $2.4 \mathrm{GHz}$ & $2.4 \mathrm{GHz}$ \\
\hline Multiplexing & FHSS & DSSS \\
\hline Outdoor range & $10 \mathrm{~m}$ & $100 \mathrm{~m}$ \\
\hline Data rate & $2.1 \mathrm{Mbps}$ & $11 \mathrm{Mbps}$ \\
\hline
\end{tabular}

\section{WiFi and Bluetooth coexistence}

Table I shows the main specification of these two radio technologies. Since both technologies share the same $2.4 \mathrm{GHz}$ band (Industrial, Scientific and Medical band), it is possible for them to interfere with each other. To minimize this interference, there exist two basic techniques: Frequency Hopping Spread Spectrum (FHSS) [9] and Direct Sequence Spread Spectrum (DSSS) [9]. FHSS allows the device to transmit with high energy in a narrow band for limited time, after which the transmission moves on to another channel. DSSS on the other hand revolves around occupying a relatively wide band with low energy. Bluetooth hops over 79 frequencies that are $1 \mathrm{MHz}$ wide. $802.11 \mathrm{~b}$ standard defines 11 possible channels that may be used. Channel hopping also may occur in WiFi, but 600 times slower than Bluetooth [10]. FHSS and DSSS together make the coexistence of WiFi and Bluetooth possible, although they do not eliminate the interference completely. As a matter of fact, the throughput decreases by about $36 \%$ [11].

\section{Proposed Algorithm}

\section{A. Message format}

The messages generated by the application may contain information about the time stamp, phone number (and even more personal data), geographic data (GPS coordinates), emergency information, message id and message priority etc. The figure 1 shows the format of the message, where the field with $*$ is optional.

- ID: message id, which is assigned when message is generated. It is used to identify messages.

- PRI: message priority. The value can be an integer between 0 and 10, which implies maximum broadcast times (TTL). For instance, priority 0 makes TTL value 1 and means that this message will be broadcast by the same node only once. The higher the priority, the 


\begin{tabular}{|c|c|c|c|c|c|c|}
\hline $\mathrm{ID}$ & $\mathrm{PRI}$ & $\mathrm{GT}$ & $\mathrm{PD}$ & $\mathrm{EI}$ & $\mathrm{GD}^{*}$ & $\underbrace{\mathrm{MD}^{*}}_{\text {Bbits }}$
\end{tabular}

Fig. 1. Emergency Message format

larger the TTL. The level of priority can be generated automatically by the application according to emergency information and modified by users if necessary.

- GT: generated time. The exact time when the message was generated.

- PD: personal data. It can contain the specific smartphone user's personal data like phone number, user's name, user's home address and so forth.

- EI: emergency information. Any important text information related to the user's emergency situation can be included such as type of the emergency, implicated emergency service, text created by the phone user and so forth.

- GD*: geographic data, the location information obtained by GPS. If the source has a GPS, then GD field will be included in the message, otherwise all the first-hop nodes could fill this field with their geographic information using their GPS.

- MD*: multimedia data. It can contain any multimedia data like photos, voice recording, or short videos to make the emergency message more vivid and informational.

- RE*: reserved field, not defined yet.

According to the above message format, it can be easily seen that the total size of the message is less than 100kbit in the absence of multimedia data. The data rate of Bluetooth and $\mathrm{WiFi}$ can be up to $2.0 \mathrm{Mbit} / \mathrm{s}$ and $11.0 \mathrm{Mbit} / \mathrm{s}$ respectively. Hence, the transmission time of the messages without additional multimedia data could be ignored. However, even if the multimedia data is included in the message (we assume that one 2MByte video included), the transmission time is still relatively short (8 seconds for Bluetooth and 2 seconds for $\mathrm{WiFi}$ ) and is acceptable in our application.

\section{B. Proposed routing algorithm}

The established ad hoc network in our application is quite different from traditional ad hoc networks. As mentioned in introduction, source and destination might never be connected to the same network at the same time. Furthermore, intermediate nodes have no knowledge of the network topology. This is different from traditional ad hoc network routing. Therefore, the design of efficient routing strategies would be more challenging in this case.

According to these characteristics, the ad hoc network in our application can be regarded as an opportunistic network [12]. Figure 2 shows a possible taxonomy of routing algorithms in opportunistic networks. Due to the fact that our application is used in the case that GSM/3G networks are completely disabled and the emergency situation cannot tolerate the long

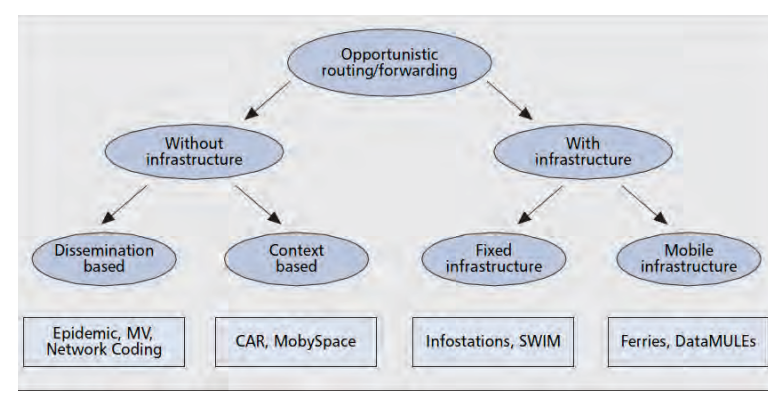

Fig. 2. Taxonomy of opportunistic routing [12]

delay, the Dissemination-Based Routing (Epidemic Routing protocol [13]) without infrastructure is well-suited for our application. However, some modifications are necessary to meet the special requirement of emergency message forwarding. In our routing approach, messages diffuse in the network by means of one-to-multiple contacts, which is similar to diseases or viruses.

A node is infected by a message when it either generates that message (in the case of the source node) or alternatively, receives it from another node. The infected node stores the message in a local buffer and broadcasts the message to find the susceptible nodes, which are those that have not yet received the message. The infection starts when susceptible nodes come into contact with an infected node and ends when it receives the message completely. It is worth mentioning that there are distinct behaviors of infected nodes with priority 0 message and those with higher priority message. An infected node with a priority 0 (lowest priority) message broadcasts this message only once and then drops it. That is to say, an infected node recovers (healed from the disease) immediately and becomes immune to the same disease forever. An infected node with higher priority message broadcasts this message $N$ times ( $N$ is the value of TTL), which means that this node will not recover until it broadcasts the same message $N$ times (no matter whether it infects any other node or not). This behavior increases the probability of successfully sending messages to the destination, which is crucial in the emergency situation.

Unlike epidemic routing protocol, in our routing protocol the message can traverse through infinite hops, because of the emergency application's mission, which does its best to send messages to the destination.

\section{SIMULATION}

\section{A. Setting and flow}

By checking the mobile phone product database of the top five worldwide mobile phone manufacturers, we learned how many phones support Bluetooth or $\mathrm{WiFi}$, together with an approximate ratio between them, which give us a hint on the simulation parameter setting. Table II shows that the ratio is approximately 1:16:10.

According to statistic data, the population density around crowded region may be 1000 persons per square kilometer. We assume that 40 percent of them possess a smartphone. 
TABLE II

SITUATION OF WIRELESS EQUIPMENT ON MOBILE PHONES

\begin{tabular}{l|rrrr}
\hline & all models & Bluetooth only & WiFi only & Both \\
\hline Nokia & 129 & 2 & 76 & 40 \\
\hline Samsung & 189 & 9 & 113 & 33 \\
\hline SonyEricsson & 60 & 0 & 44 & 13 \\
\hline Motorola & 62 & 3 & 29 & 27 \\
\hline Apple & 5 & 0 & 0 & 5 \\
\hline Total & 511 & 17 & 273 & 168 \\
\hline
\end{tabular}

Hence there will be about 100 nodes in a simulation region of 0.25 square kilometer.

As Figure 3 shows, the simulation starts by placing Starting Nodes somewhere (random or exact center) within the simulated disaster area. This region is surrounded by a normal region with destination. Both of the mentioned areas are rectangular in shape for the purpose of simplicity. The simulation has two ending conditions: either the message is moved outside of the emergency region or simulation has reached the maximum number of iterations.

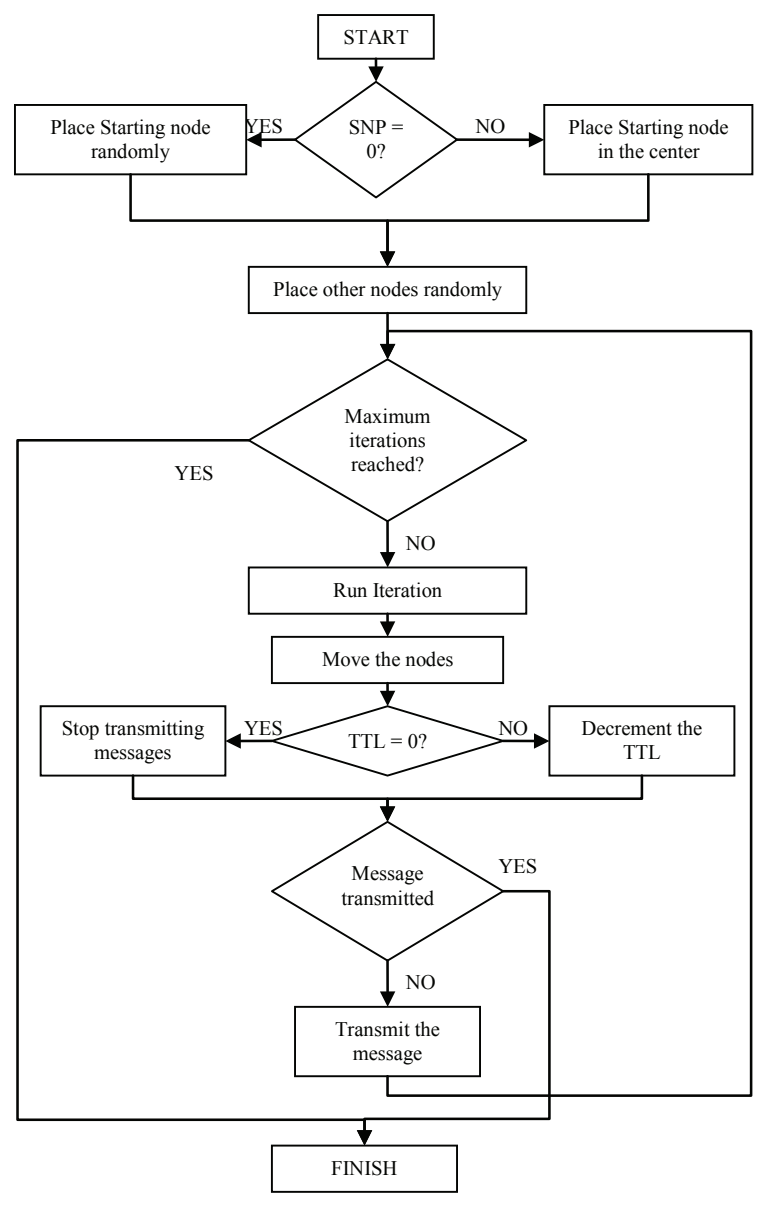

Fig. 3. Flowchart of simulation

\section{B. Script and parameters}

For the purpose of the research, two tools were created using Matlab: 1) a graphical interface to observe the outcome of a single simulation step by step to make sure that the model is behaving properly under different circumstances; 2 ) a simulation script for running thousands of simulations in an automated manner. The aim of the simulation script is to allow us to get a large sample space in order to evaluate our model more precisely.

The simulation script accepts several parameters, which are listed below.

- Area - side length of the disaster area in meters

- RangeBT - range of the Bluetooth transmission in meters, set as 10 meters.

- RangeWI - range of the WiFi transmission in meters

- MaxMove - maximum movement offset allowed for a node in meters per step.

- Probability - probability of a node to be disconnected

- TTL - number of steps after which the node will stop transmitting since it received the message

\section{Node activities}

Intermediate nodes can perform the following actions.

1) Moving: Every step the node's $x$ and $y$ coordinates are updated by a random offset from the interval: (-MaxMove, MaxMove), meaning that the node can move both up/down and left/right in relation to its initial position.

2) Message dissemination: During every step the nodes carrying the message look for nodes without the very message to send it. This process is described in epidemic routing protocol as this: infected nodes broadcast the virus (the message) to find the susceptible nodes not yet being infected by this virus. The determination whether a pair of nodes are able to participate in the transmission is based on the calculation of Euclidean distance between them as the equation 1 shows.

$$
d(p, q)=\sqrt{\left(p_{1}-q_{1}\right)^{2}+\left(p_{2}-q_{2}\right)^{2}}
$$

where $p_{1}$ and $p_{2}$ are the coordinates of the node sending the message and $q_{1}$ and $q_{2}$ are the coordinates of the receiving node.

3) Immune to the same message: After a node receives a message, its internal TTL counter is set to a value $(N>0)$. After each iteration this value is decreased by one. Once the counter reaches 0 , the node discards the message and stops taking part in the peer to peer broadcasting.

4) Disconnecting from the network: Every intermediate node might be switched-off during the dissemination in an ad hoc network, thus it is reasonable for every node to randomly stop participating in the message broadcast.

\section{RESULTS AND FINDINGS}

Figure 4 shows an example of running Graphic User Interface. Using the test script, we run every test case 10000 times and get raw testing data.

Since in reality it is impossible to affect some of the factors discussed below like the disaster area, the number and range of 


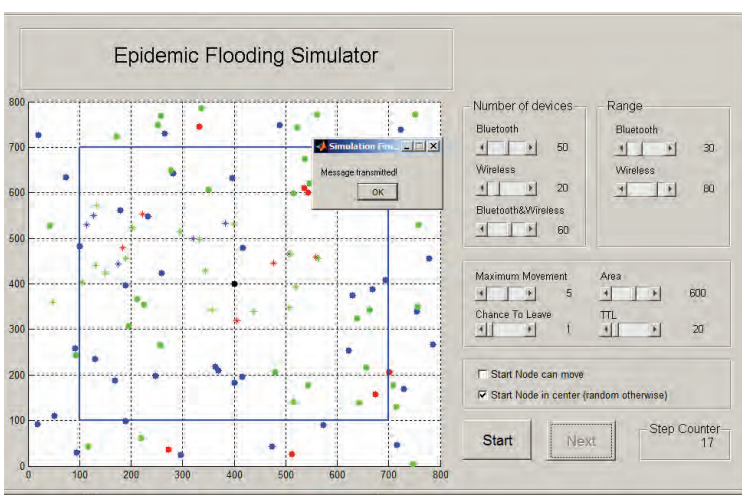

Fig. 4. Developed Graphic User Interface

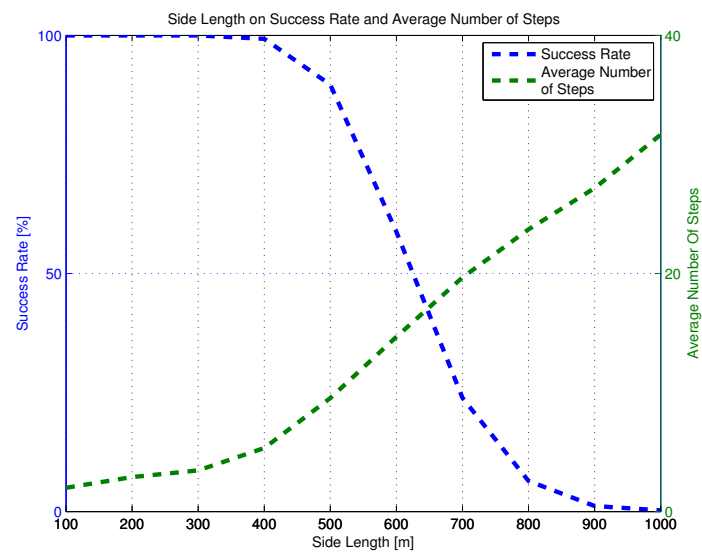

Fig. 5. Influence of disaster area side length

the wireless devices, therefore more attention should be paid to tunable factors such as TTL value.

Starting with the influence of the Area Side Length on the simulation outcome from Figure 5, it can be observed that relatively small lengths (100, 200 and 300 meters) combined with WiFi Range of $100 \mathrm{~m}$ produce $100 \%$ success rate. However, beginning at the value of 500 meters a significant drop can be noticed and a following increase in the Average Number of Steps (from less than 10 to over 20) does not compensate this decrease of success rate.

Moving on to $\mathrm{WiFi}$ range in Figure 6 , it has a quite significant influence on the success rate. $75 \%$ success rate with range 90 meters sharply drops to $25 \%$ with 60 -meter range. WiFi range getting less and less optimistic, the increase of average number of steps (from 20 to more than 30) does not cancel out the negative effect of lowering the WiFi range. To make the system useful, the average outdoor WiFi range in practice should be kept above 80 meters. As a consequence, a relatively good communication environment (with low channel noise/interference and few large obstacles) would be beneficial on the wireless transmission range, which guarantees the chance of successful message dissemination.

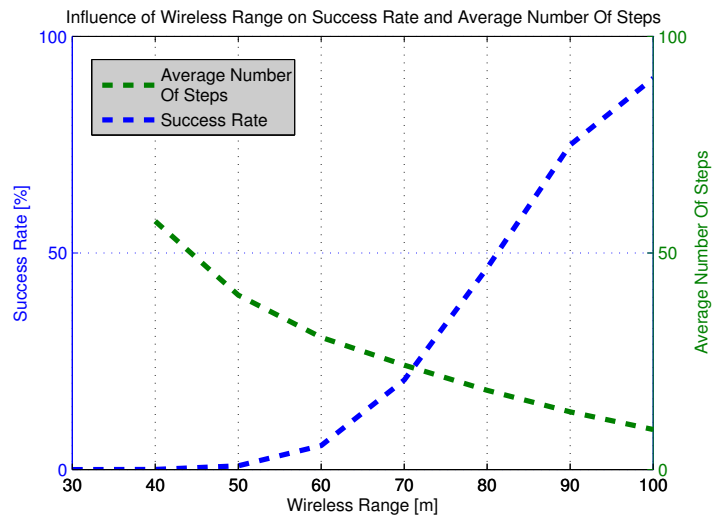

Fig. 6. Influence of WiFi range

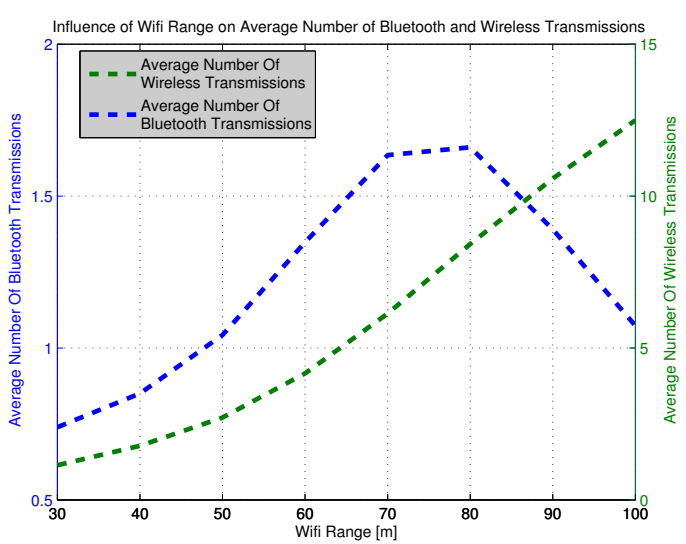

Fig. 7. Bluetooth and WiFi's contribution with WiFi range

As a side note, when we look at figure 7, the influence of WiFi range on the Average Number of Bluetooth and WiFi transmissions, we can see that, while initially both values increase with WiFi Range, at 80 meters Bluetooth Transmissions start to drop, while the Wireless ones continue to raise. And all the test cases show that WiFi plays a major role in the message dissemination, while Bluetooth plays a supporting role, due to its relatively short range (although partly compensated by higher density). Hence, the whole emergency system is more dependent on the WiFi technology.

Next, one can see that from Figure 8 that for relatively low values of TTL (in the range from 1 to 20), which stands for lower priority, significant positive correlation can be observed. Within this interval the success rate can change from around $57 \%$ to more than $90 \%$. However, increasing this value further does not introduce a significant improvement. It is obvious that to ensure higher success rate calls for more steps/time and more smartphone participation. Thus, it is vital to strike a balance between the success rate and cost. The red curve stands for performance-cost ratio (success rate divided by average number of steps), which means the whole transmission efficiency at the cost of time and smartphone energy 


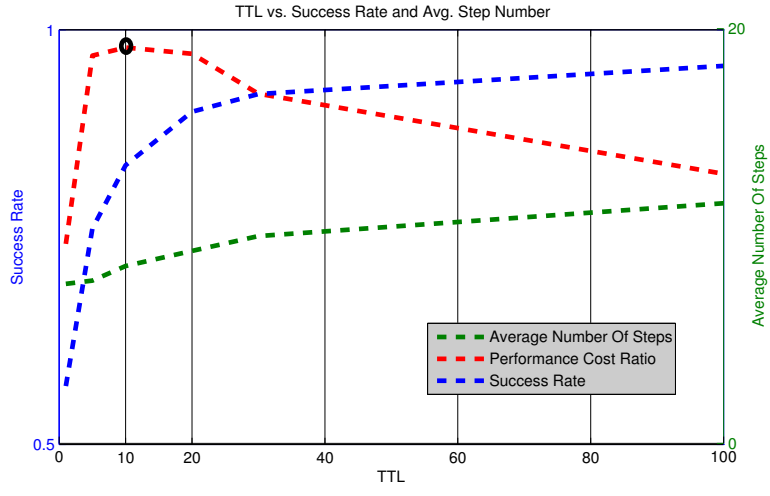

Fig. 8. Influence of TTL/priority on performance

consumption. At the peak point (where TTL=10), the optimal trade-off is achieved. So when implementing our application, it is reasonable to set $T T L=10$ as the default parameter. Hereby, simulation result provides a valuable baseline of priority setting that could be used for the most common cases.

When we look at the data produced from changing the Probability value, we can see that increasing this value by $1 \%$ continuously lowers the success rate by about $7 \%$ each time. Thus, prolonging every smartphone's operational hours in micro level could improve the success rate in macro level.

Next, Maximum Movement does have significant influence (as even setting it to 0 (static) produced a positive outcome in $48 \%$ of the simulations), and beyond the value of 8 , the result do not change greatly.

Lastly, setting everything else to default values, while allowing the source node to move during the simulation gives only slight improvement over the option to make it static (about $3.4 \%$ ). In other words, a static source does not degrade the system performance much.

\section{CONCLUSION AND FUTURE WORK}

In this paper we explored the usage of smartphones as message disseminating nodes that do not depend on cellular infrastructures. Our application is useful during natural hazards, when base stations are destroyed, rendering the phone users unable to call or send a SMS to other users. Under such circumstance, we proposed an emergency message dissemination system by taking advantage of epidemic routing algorithm, as well as Bluetooth and WiFi technologies present in modern smartphones.

For the purpose of verifying our research, we have simulated the system with two tools: Epidemic Routing Simulator with graphical user interface, and a simulation script. We tested the influence of various parameters (disaster area, number of devices, range of devices, node movement speed, chance of a node to drop a connection, message priority) on the success rate of the message delivery function.

Based on the data gathered from the second tool, we can conclude that among all of the investigated parameters the ones with the significant influence on the probability of a successful message transmission out of the disaster area are the Node Density, WiFi Devices' Range, Message Priority and Probability of Node Leaving the Network. Regarding the wireless technologies, the result shows that WiFi makes much greater contribution than Bluetooth on message dissemination. Furthermore, a trade-off should be made between success rate and average time/energy cost. The simulated results also provide a useful baseline for practical parameter setting in performance-cost optimization.

On the other hand, whether the source node is dynamic or static is not a big factor in relation to the success rate, which is a good news for trapped victims. Based on the analysis, we suggest other positive approaches, such as improving the wireless transmission range and improving the battery life to prolong smartphone participation in the system.

Possible future work includes more realistic modeling of node movement, instead of simple random model in current implementation, and extending the model to allow two-way message exchange that would enable the system to perform peer to peer routing, instead of relying on epidemic flooding, so as to achieves reliability. Last but not least, multi-message simulation could be carried out to test proposed routing algorithm.

\section{REFERENCES}

[1] H. Uwe, Pervasive Computing: The Mobile World. Springer, 2003.

[2] comScore, "U.s. mobile subscriber market share," Press Release, 2010.

[3] Z. Epstein, "Smartphone shipments grew 74\% in 2010," Berg Insight, 2011.

[4] R. Ballagas and J. Borchers, "The smart phone: a ubiquitous input device," Pervasive Computing, vol. 5, pp. 70-77, 2006.

[5] Z. Hassan, "Ubiquitous computing and android," Digital Information Management, vol. 2, pp. 166 - 171, 2008.

[6] G. Bai, L. Gu, and T. Feng, "Context-aware usage control for android," Security and Privacy in Communication Networks, vol. 50, pp. 326-343, 2010.

[7] J. Therese and B. Fajardo, "A mobile disaster management system using the android technology," WSEAS TRANSACTIONS on COMMUNICATIONS, vol. 9, 2010

[8] J. Haartsen, "Bluetooth: The universal radio interface for ad hoc, wireless connectivity." Ericsson Review, vol. 3, 1998.

[9] J. H. Schiller, Mobile Communications. Addison-Wesley, 2000.

[10] J. Lansford, A. Stephens, and R. Nevo, "Wi-fi (802.11b) and bluetooth: enabling coexistence," IEEE network, vol. 15, 2001.

[11] L. Ophir, Y. Bitran, and I. Sherman, "Wi-fi (ieee 802.11) and bluetooth coexistence: Issues and solutions," IEEE International Symposium on Personal Indoor and Mobile Radio Communications, vol. 2, p. 847C852, 2004.

[12] L. Pelusi and rea Passarella, "Opportunistic networking data forwarding in disconnected mobile ad hoc networks," IEEE Communications Letters, 2006.

[13] A. Vahdat and D. Becker, "Epidemic routing for partially connected ad hoc networks," Tech. Rep. CS- 2000-06, Department of Computer Science, Duke University, 2000. 